\title{
Studies on the Structural Abnormality of Fibrinogen Paris I
}

\author{
Michael W. Mosesson, David L. Amrani, and Doris Ménaché \\ From the Department of Medicine of the State University of New York, \\ Downstate Medical Center, Brooklyn, New York 11203 and Service Central \\ d'Immunologie et Hématologie, Hôpital Beaujon, 92110 Clichy, France
}

A в S T R A C T The structural properties of an inherited fibrinogen abnormality designated fibrinogen Paris I were investigated. Dodecyl sulfate gel electrophoresis of unreduced samples revealed no discernible differences in molecular weight from normal; this implied that in fibrinogen Paris I, the normal fibrinogen architecture of six covalently linked chains per molecule is preserved. Examination of dithiothreitol reduced samples before and after treatment with Reptilase or thrombin revealed that the $\mathrm{A} \alpha$ - and $\mathrm{B} \beta$-chains could release the $\mathrm{A}$ and $\mathrm{B}$ peptides, respectively. A mutant chain (mol wt 52,500, termed $\gamma$ Paris I) which replaces a large proportion of $\gamma$-chains (mol wt 49,400) was shown, like normal $\gamma$-chains, to lack thrombin- and Reptilase-sensitive sites. The $\gamma$-chains and $\alpha$-chains of Paris I fibrin underwent Factor XIIIa-catalyzed cross-linking slowly; this behavior was not attributable to an intrinsic abnormality of these chains themselves but rather to the inhibitory effect of the mutant $\gamma$ Paris I chains on this process. Results of DEAE-cellulose gradient elution chromatography of Paris I fibrinogen preparations revealed the presence of small amounts of normal fibrinogen molecules and also indicated that the $\gamma$ Paris I chains possessed structural overlap with $\boldsymbol{\gamma}$-chains. Unlike $\boldsymbol{\gamma}$-chains however, the $\gamma$ Paris I chains did not incorporate dansylcadaverine in the presence of Factor XIIIa, nor, as previously reported, did they undergo cross-linking. The observations indicate that the amine acceptor site found in the $\mathrm{COOH}$ terminal region of the $\gamma$-chain is either not present on the $\gamma$ Paris I chain or is unavailable for cross-linking. Further support for localization of the abnormality in the $\mathrm{COOH}$-terminal region of the molecule was obtained from the observation that during plasmic hydrolysis of Paris I fibrinogen, at least one unique form of core Fragment D (DParis I) was evolved, whereas Fragment E did not differ from normal.

This work was presented in part at the 18th Annual Meeting of the American Society of Hematology, 9 December 1975 .

Received for publication 2 July 1975 and in revised form 30 October 1975.

\section{INTRODUCTION}

In 1963 Ménaché (1) described an inherited abnormality of plasma fibrinogen which has come to be known as fibrinogen Paris I (for recent comprehensive reviews on this general subject see references 2 and 3 ). The functional defect is featured by a marked prolongation of the thrombin time (1) which is evidently due to delay of the aggregation phase of fibrin derived from such plasma (4). An additional important characteristic is its profound ability to delay the aggregation phase of normal fibrin as well (4). In this regard, in terms of its ultimate ability to form a clot, at least two subpopulations of Paris I fibrinogen have been recognized (4).

Biochemical analyses suggest that the $\mathrm{NH}_{2}$-terminal amino acids and thrombic peptides $\mathrm{A}$ and $\mathrm{B}$ of fibrinogen Paris I are qualitatively the same as those of normal fibrinogen $(2,5)$. Furthermore, no abnormalities of that portion of the A $\alpha$-chain of fibrinogen Paris $\mathrm{I}$ isolated with the $\mathrm{NH}_{2}$-terminal disulfide knot have been found $(5,6)$. Preliminary results of gradient elution ion-exchange chromatography on DEAE-cellulose (7) indicate that fibrinogen Paris I has a greater anionic-binding capacity than does normal fibrinogen. Most recently, results of dodecyl sulfate gel-electrophoretic analyses of reduced fibrin clots of Paris I fibrinogen have revealed the presence of an abnormal chain that is somewhat larger than the $\gamma$-chain (8). Since densitometric scans indicate that it replaces the normal $\boldsymbol{\gamma}$-chain, it has been termed $\gamma$ Paris I, although it does not participate in fibrin cross-linking as do normal $\gamma$-chains (8).

In the studies to be reported, we present experiments which extend those cited above. They permit additional conclusions to be drawn with respect to structural and functional properties of the fibrinogen Paris I molecule, and provide evidence that the defect in the $\gamma$ Paris $I$ chain is localized in its $\mathrm{COOH}$-terminal region.

\section{METHODS}

Chromatographic analysis. DEAE-cellulose (Whatman DE-23) column chromatography of unmodified samples was 
carried out at $2^{\circ} \mathrm{C}$ on $0.9 \times 30-\mathrm{cm}$ columns employing a combined $\mathrm{pH}$ and phosphate gradient from $0.005 \mathrm{M}$ phosphate, $\mathrm{pH} 8.6$ to $0.50 \mathrm{M}$ phosphate, $\mathrm{pH} 4.1$ to $4.3(9,10)$. Fractions corresponding to $1 \%$ of the total gradient were collected.

Electrophoretic and related procedures. Dodecyl sulfatepolyacrylamide gel-electrophoresis was performed essentially as described by Weber and Osborn (11) in gels whose final acrylamide concentration was 5 or $9 \%$. In all figures showing results of such experiments, the gels are aligned with the anode at the bottom of the figure. For disulfide bond reduction, when desired, dithiothreitol ( $14 \mathrm{mM}$, final concentration) was employed. Gels were stained with amidoSchwartz 10B and then counterstained with Coomassie Brilliant Blue (12). Densitometric scans of stained gels were made in a Gilford model 240 spectrophotometer (Gilford Instrument Laboratories, Inc., Oberlin, Ohio) equipped with a Linear Transport apparatus. Determination of the radioactivity of the stained bands of radioiodinated gel samples (5\% acrylamide) was accomplished by slicing out the bands (approximately 2-3-mm slices) with a razor blade and then counting their radioactivity in a Picker Autowell II gamma counter (Picker Corp., Cleveland, Ohio).

Protein markers used were the various fibrinogen subunit chains and their derivatives whose molecular weights had been determined $(12,13)$ by calibration against standard marker proteins. Identification of chains retaining peptide A or B was made by comparison of the mobility of reduced samples in dodecyl sulfate electrophoresis before and after treatment with Reptilase (Pentapharm, Ltd., Basel, Switzerland) or thrombin (lot H-1, provided by Dr. D. L. Aronson, Bureau of Biologics, Food and Drug Administration) as previously described (12).

Immunoelectrophoresis (14) and agarose (Behring Diagnostics, Somerville, N. J.) electrophoresis (1\% agarose) were carried out in a standard manner $(12,13)$ with rabbit antihuman fibrinogen serum and rabbit antihuman coldinsoluble globulin serum (9). Electroimmunoassay (15) for determination of the plasma fibrinogen level was performed as previously described (9).

Radioactive labeling procedures. Normal fibrinogen fraction I-4 and fibrinogen Paris I (chromatographic peak 1) were radioiodinated with ${ }^{125} \mathrm{I}$ (carrier free, New England Nuclear, Boston, Mass.) by the iodine monochloride method of McFarlane (16). Labeling efficiency was $30-50 \%$. The iodine content of the labeled fibrinogen was less than 0.5 atom/molecule. After the labeling procedure, as suggested by the studies of Krohn et al. (17), the radioiodinated fibrinogen sample was diluted by adding "cold" fibrinogen in an amount equal to that in the labeling mixture. Extensive dialysis against $0.3 \mathrm{M} \mathrm{NaCl}$ was then carried out. The labeled protein was stored at $-20^{\circ} \mathrm{C}$.

Preparation of fibrinogen for analysis. Since Paris I fibrinogen clots poorly, assays based upon recovery of a clot from plasma (18) grossly underestimate its actual level (i.e. levels determined in this study were approximately $0.3 \mathrm{mg} / \mathrm{ml}$, normal range $1.8-4.0 \mathrm{mg} / \mathrm{ml}$ ). A more accurate measurement of the fibrinogen level of Paris I plasma samples and the amount of fibrinogen precipitated in Cohn ethanol fraction I was made possible by electroimmunoassay (15) before and after removal of Cohn fraction I (19). After fractionation of one such plasma (fibrinogen level $3.88 \mathrm{mg} / \mathrm{ml}$ ) $81 \%$ of immunoassayable material had been precipitated with Cohn fraction I; this value is within the range found upon fractionation of normal plasma fibrinogen (viz., 70-88\%). However, it does not appear that the

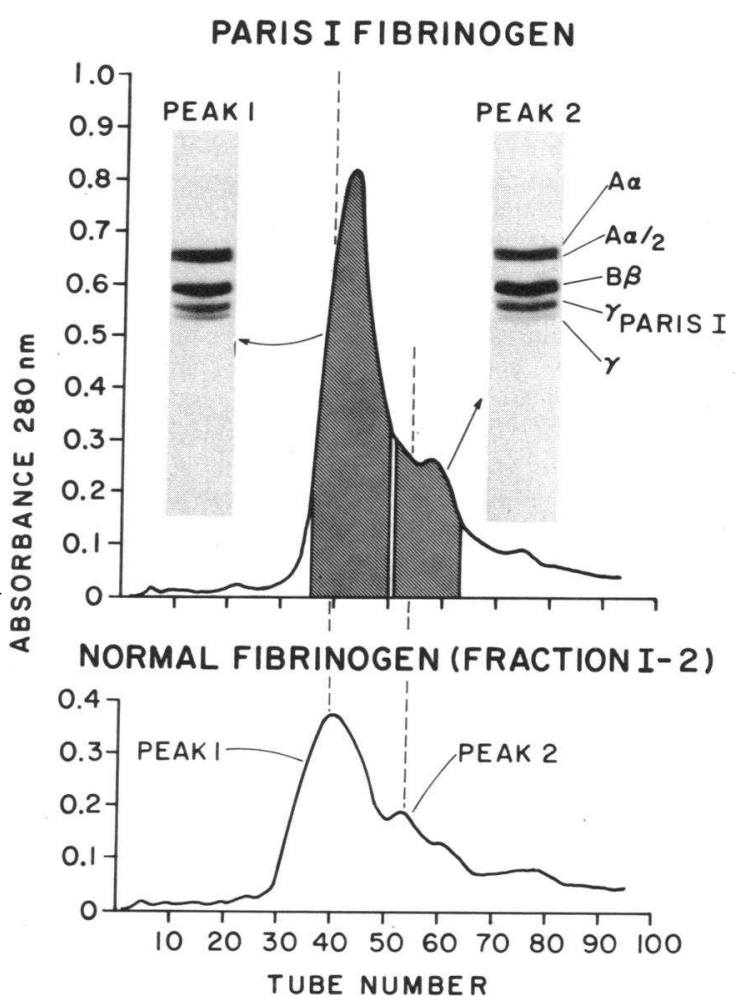

FIGURE 1 DEAE-cellulose gradient elution chromatogram of $54 \mathrm{mg}$ fibrinogen Paris I fraction I-2 (upper panel) and $19 \mathrm{mg}$ normal fibrinogen fraction I-2. Chromatograms were developed separately under identical conditions. Fractions were pooled as shown by the hatched areas. The electrophoretic band pattern of reduced samples in 9\% acrylamide gels containing dodecyl sulfate is shown for the peak 1 and 2 pools, respectively.

subtle differences in solubility which have been reported (20) could be discerned by this type of analysis.

Fraction I, obtained on three occasions from two samples of Paris I plasma, was extracted twice with the glycinecitrate-ethanol buffer described by Blombäck and Blombäck (21) to prepare fraction I-0. This material was then subfractionated into fractions I-1 and I-2 by the procedure of Laki (22). The mean yield of protein in fraction I-2 (0.7, $0.9,1.3 \mathrm{mg} / \mathrm{ml}$ starting plasma, respectively) was in the same range found for two control samples of normal plasma ( 0.8 and $1.1 \mathrm{mg} / \mathrm{ml}$ plasma, respectively). The coagulability (23) of Paris I fraction I-2 was considerably lower (mean $=55 \%$, range $=49-60 \%$ ) than that found for normal fibrinogen fraction I-2 (92-95\%, respectively). The Paris I "clot" whch did form appeared as a fine to medium granular precipitate. Nevertheless, agarose electrophoresis and immunoelectrophoresis indicated that purification of Paris I fraction I-2, comparable to that for normal fraction I-2, had been achieved. That is, for both samples, agarose electrophoresis demonstrated a major fibrinogen band (confirmed by immunoelectrophoresis) and a small amount of a faster migrating band. This latter band was identified by immunoelectrophoresis as "cold-insoluble globulin" (9), a nonfibrinogen component of the fraction. DEAE-cellulose gradient elution chromatography (Fig. 1) was employed 
to characterize the fibrinogen itself as well as to provide a means for additional purification. Eluate fractions were pooled in two batches corresponding to the two major fibrinogen-containing chromatographic peaks (24) (peaks 1 and 2 , respectively), concentrated by precipitation of protein at $33 \%$ saturated $\left(\mathrm{NH}_{4}\right)_{2} \mathrm{SO}_{4}$, redissolved in $0.3 \mathrm{M}$ $\mathrm{NaCl}$, dialyzed against the same solution, and stored at $-80^{\circ} \mathrm{C}$ until further use. Analytical procedures were carried out with the peak 1 pool unless otherwise indicated.

Normal human fibrinogen fraction I-4 was prepared by standard procedures as previously described (23).

Cross-linking of fibrin. Dilution of fibrinogen samples and other components of the clotting mixture were made with $0.1 \mathrm{M}$ Tris- $\mathrm{HCl}$ buffer, $\mathrm{pH}$ 7.5. The final concentration of the components of the cross-linking mixture were: thrombin 0.4 U. S. U/ml, Factor XIII, 36 Loewy (25) U/ $\mathrm{ml}$; $\mathrm{Ca}^{++}, 12 \mathrm{mM}$; cysteine $\mathrm{HCl}$ (adjusted to $\sim \mathrm{pH} 7$ with Tris), $12 \mathrm{mM}$; fibrinogen, 0.6 or $1.2 \mathrm{mg} / \mathrm{ml}$ (in mixtures of Paris I and normal fibrinogen). Human plasma Factor XIII (26) was a generous gift from Dr. S. I. Chung, National Institute of Dental Research, Bethesda, Md. The clotting mixture was incubated for $18 \mathrm{~h}$ at room temperature before terminating the cross-linking reaction and solubilizing the clot by adding an equal volume of a dithiothreitol-urea-sodium dodecyl sulfate $(2 \% / 10 \mathrm{M} / 2 \%)$ solution. Portions of this solution were then subjected to dodecyl sulfate gel electrophoresis.

Fluorescent amine incorporation into fibrin. Factor XIIIacatalyzed incorporation of the fluorescent amine, dansylcadaverine (dansylcadaverine sulfate, a gift from Dr. Rolf Huseby), into acceptor sites on fibrin (27) was carried out under the general conditions for cross-linking of fibrin described above. The final concentration of Factor XIII was 4 Loewy (25) $\mathrm{U} / \mathrm{ml}$; monodansylcadaverine, $9 \mathrm{mM}$. Inasmuch as peak 1 Paris I fibrinogen was no longer available at the time this particular experiment was carried out chromatographic peak 2 of Paris I fibrinogen was used at a final concentration of $1 \mathrm{mg} / \mathrm{ml}$. The Paris I clots which formed were centrifuged, dialyzed against $0.1 \mathrm{M}$ Tris- $\mathrm{HCl}$ buffer, pH 7.4 containing $0.01 \mathrm{M}$ EDTA (Tris-EDTA-Cl) and then dissolved with an equal volume of dithiothreitolurea-sodium dodecyl sulfate solution for electrophoretic analysis. After electrophoresis, gel samples were fixed for $2-3 \mathrm{~h}$ in $10 \%$ trichloroacetic acid (wt/vol), equilibrated against several changes of $0.1 \mathrm{M}$ Tris- $\mathrm{HCl}$ buffer, $\mathrm{pH} 7.4$ and examined under UV light for fluorescence. Selected gels were also stained with Coomassie Brilliant Blue to be assured of the identity of each fluorescent band and of the presence of substantial amounts of the $\gamma$ Paris I band in the case of fibrinogen Paris I samples.

Plasmic hydroly'sis of fibrinogen. ${ }^{1}$ This procedure was carried out in $0.05 \mathrm{M}$ Tris- $\mathrm{HCl}$ buffer, $\mathrm{pH} 8.6$ at $37^{\circ} \mathrm{C}$.

${ }^{1}$ The terminology applied in this study to plasmic hydrolysis is based upon that developed previously $(12,13)$. The degradative phases of plasmin hydrolysis of fibrinogen have been divided into stages 1,2 , and $3(28)$. Stage 1 is characterized by the presence of coagulable species; stage 3 , by core fragments containing either $D$ or $E$ antigenic determinants, but not both. In stage 2 , there exist core species with both $\mathrm{D}$ and $\mathrm{E}$ antigenic determinants, but no (or relatively few) coagulable species.

Terminology for the plasmic core fragments $D$ and $E$, identifiable in intermediate and advanced digests, is the same as that proposed by Nussenzweig et al. (29). Forms of Fragments $\mathrm{D}$ and $\mathrm{E}$ arising in digests of normal fibrinogen, distinguishable by their electrophoretic migration in the
The final protein concentration (normal or Paris I fibrinogen) was $1 \mathrm{mg} / \mathrm{ml}$. Plasmin ( 10.2 caseinolytic $\mathrm{U} / \mathrm{ml}$ ) had been prepared at the Michigan Department of Health (33). It was used at final concentrations varying from 0.06 to $1.0 \mathrm{U} / \mathrm{ml}$. This range of enzyme concentration permitted examination of samples at all phases of digestion. Sampling of the digest was accomplished by transferring a portion to an equal volume of sodium phosphate $(0.02 \mathrm{M})$ buffer, $\mathrm{pH}$ 7.0 , containing $8 \mathrm{M}$ urea and $2 \%$ sodium dodecyl sulfate.

\section{RESULTS}

Molecular size and subunit structure of Paris I fibrinogen. Comparison of unreduced samples of Paris I fibrinogen with normal fibrinogen revealed no discernible differences in their anodal migration rates in sodium dodecyl sulfate-containing gels (Fig. 2). This indicated that Paris I fibrinogen had a molecular size which was the same (or nearly so) as that of normal ${ }^{2}$ and implied that the essential fibrinogen architecture (six covalently linked chains per molecule) had been preserved. The Paris I sample was resolved into two major bands corresponding in anodal migration rate to bands I and II of normal fibrinogen. Recent studies with normal human fibrinogen have characterized band II as containing intermediate catabolic species characterized by loss of $\mathrm{COOH}$-terminal portions of A $\alpha$-chains (31). This therefore suggests that Paris I fibrinogen undergoes similar catabolic processes to those which have been identified for normal fibrinogen $(12,23,31)$.

Treatment of fibrinogen samples with Reptilase or thrombin followed by reduction and dodecyl sulfate gelelectrophoretic analysis, permits a functional identifica-

presence of dodecyl sulfate, are indicated by numerical subscripts; the higher the number, the smaller the fragment (e.g., $D_{1}$ through $D_{5}$ ). A Fragment $D$ band unique to Paris I fibrinogen digests is termed DParis I. Core species larger than Fragment D, identifiable by their migration as bands in sodium dodecyl sulfate-containing gels, have been designated with roman numerals corresponding to decreasing size (i.e. I, largest; II, next largest; and so forth), as suggested by Mills and Karpatkin (30). Digest bands VI and VII (31) appear to correspond to the band identified as Fragment $\mathrm{X}$ by Marder et al. (32). Band VIII corresponds to Fragment $\mathrm{Y}$.

The intact subunit chains of fibrinogen are designated $\mathrm{A} \alpha, \mathrm{B} \beta$ and $\gamma$; the mutant chain replacing the normal $\gamma$-chain in Paris I fibrinogen is termed $\gamma$ Paris I. Cleavage at sites other than those attacked by thrombin is indicated by a diagonal line on the $\mathrm{COOH}$ - or $\mathrm{NH}_{2}$-terminal side. Thus $\mathrm{A} \alpha \rightarrow \mathrm{A} \alpha /+/ \alpha ; \mathrm{B} \beta \rightarrow \mathrm{B} \beta /+/ \beta$, and so forth. Individual chain fragments are further designated with a numerical subscript $(12,13,31)$.

${ }^{2}$ Increases in the size of core species resulting from the substitution of $\gamma$ Paris I mutant chains for $\gamma$-chains are probably too small to be distinguished in unreduced samples. For example, the difference between unmodified fibrinogen ( $\mathrm{mol} \mathrm{wt} \sim 340,000)$ and fibrin $(\mathrm{mol} \mathrm{wt} \sim 334,000)$ under such electrophoretic conditions, is very difficult to appreciate. 


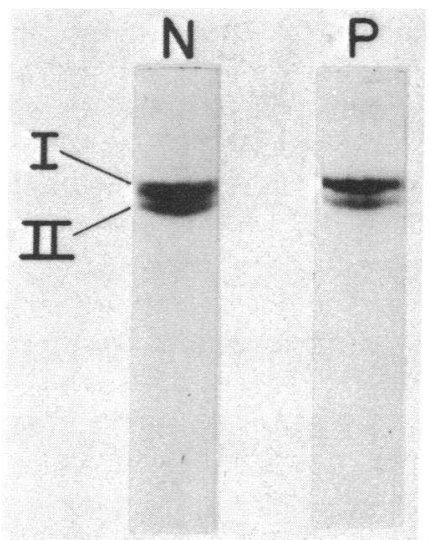

FiguRE 2 Dodecyl sulfate electrophoresis (5\% acrylamide) of unmodified samples of normal fraction I-4 $(\mathrm{N})$ and Paris I fibrinogen (P) from the chromatographic peak 2 pool. Material from the peak 1 pool was analyzed in another experiment and yielded the same band pattern. The two major fibrinogen bands ( $I$; II) found in fraction I-4 (31) are indicated.

tion of $\mathrm{A} \alpha$ - and $\mathrm{B} \beta$-chains (12). That is, $\mathrm{A} \alpha$-chains or their catabolic remnants containing peptide $A(A \alpha /)$, after treatment with Reptilase or thrombin, display a measurable molecular weight reduction (i.e. increase in anodal migration) of approximately 2,000 reflecting release of peptide $\mathrm{A}$ from the parent chain. B $\beta$-chains exhibit an increased anodal migration reflecting the release of peptide $B$, but only after thrombin treatment; $\gamma$-chains which lack a thrombin- or Reptilase-sensitive site, display this by their failure to change their migration rate after treatment with either enzyme.

Paris I and normal fibrinogen were examined after treatment with Reptilase and thrombin (Fig. 3). By the criteria outlined above, Paris I fibrinogen possessed $\mathrm{A} \alpha-, \mathrm{B} \beta-, \quad \gamma$-chains, and $\mathrm{A} \alpha /$ catabolic remnants (e.g. $A \alpha / 2$ plus other more faintly stained remnants such as $A \alpha / \vartheta)$, which were indistinguishable from those of normal fibrinogen. However as reported by Budzynski et al. (8), the $\gamma$-chains were markedly reduced in amount relative to $A \alpha$ - and $B \beta$-chains. This observation plus the presence of an additional band ( $\gamma$ Paris I; mol wt 52,500 \pm 700 ) indicated that $\gamma$-chains (mol wt 49,400, reference 13) had been replaced by a mutant chain. Like a $\gamma$-chain, the $\gamma$ Paris I band did not change its migration rate after Reptilase or thrombin treatment. Assessment of band distribution of stained gels by densitometric scanning also indicated that mutant $\gamma$ Paris I chains had replaced $\gamma$-chains, since the $\boldsymbol{\gamma}+\boldsymbol{\gamma}$ Paris I area approximated that found for the $\gamma$-chain position of normal fibrinogen.

The relatively close spacing of the $\gamma$ Paris I and $\gamma$-bands resulted in a complex densitometric peak, the components of which were not well resolved by this technique; nevertheless, it was possible to calculate ap- proximately the proportion of the $\gamma$ Paris I mutant chains relative to the $\boldsymbol{\gamma}$-chain population of normal fibrinogen by visual correction for peak overlap. For material from chromatographic peak 1 (Fig. 1) the value obtained was $65-75 \%$; for chromatographic peak 2 fibrinogen, 67$82 \%$; for plasma subfraction I-0, $60-70 \%$; for plasma subfraction I-1, 73-78\%.

Cross-linking of fibrin catalyzed by Factor XIIIa. Cross-linking of fibrin in the presence of Factor XIIIa results in the introduction of $\epsilon$ - $(\gamma$-glutamyl) lysine bridges $(34,35)$ between $\boldsymbol{\gamma}$-chains $(\boldsymbol{\gamma}$-dimer) and $\alpha$-chains ( $\alpha$-polymer) of neighboring molecules (36-39). The capacity of Paris I fibrin to form such bridges was tested by supplementing a Paris I sample with an excess of Factor XIII before addition of $\mathrm{Ca}^{++}$and thrombin. Comparable supplements were added to normal fibrinogen fraction I-4 (Fig. 4), which was then subjected to crosslinking under identical conditions. At a time when virtually complete cross-linking of normal fibrin had occurred, only $\gamma$-chains of Paris I fibrinogen had undergone cross-linking. Small amounts of monomeric $\boldsymbol{\gamma}$-chain were still evident, and there was no evidence of $\alpha$-polymer formation. In other experiments (not shown) complete $\boldsymbol{\gamma}$-dimerization of the $\boldsymbol{\gamma}$-chains in Paris I fibrin was obtained as well as some degree of $\alpha$-polymer formation. Under no circumstance was there ever any indication that the $\gamma$ Paris I mutant chain had participated in the crosslinking process.

Mixtures of normal and Paris I fibrin (Fig. 4, gel 3) showed incomplete $\boldsymbol{\gamma}$-dimer formation, and minimal $\alpha$-polymer formation. Determination of the radioactivity of gel slices from a mixture of ${ }^{125} \mathrm{I}$-labeled normal fibrin and unlabeled Paris I fibrin indicated that at a time when normal fibrin should have undergone complete $\gamma$-dimerization (e.g., gel 1 ), only $55 \%$ of the ${ }^{125}$ I-labeled $\gamma$-chain

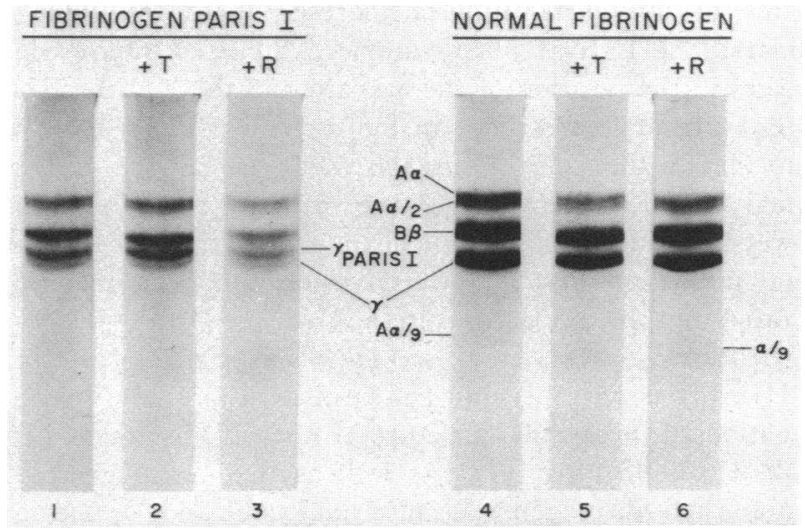

Figure 3 Dodecyl sulfate electrophoresis (9\% acrylamide) of normal fibrinogen fraction I-4 and Paris I fibrinogen before and after treatment with Reptilase (R) or thrombin ( T). 


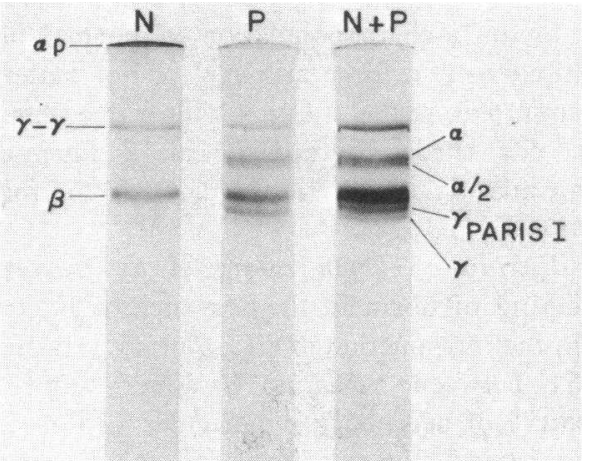

FIGURE 4 Dodecyl sulfate electrophoresis (9\% acrylamide) of reduced samples of normal fibrin (N), Paris I fibrin $(\mathrm{P})$, and a mixture of equal parts of normal and Paris I fibrin $(\mathrm{N}+\mathrm{P})$ in the presence of Factor XIIIa under identical cross-linking conditions. The same electrophoretic result was obtained with radioactively labeled material.

population had formed $\gamma$-dimers. Thus, when cross-linked in the presence of Paris I fibrin, the cross-linking of normal fibrin is grossly impaired.

Cross-linking in the presence of dansylcadaverine. Since the $\gamma$ Paris $I$ chain does not participate in crosslinking, this mutant may also lack the glutamine acceptor site normally available on $\gamma$-chains (36). This possibility was explored by examining the reduced gels of normal and Paris I fibrin after they had been subjected to the action of Factor XIIIa in the presence of monodansylcadaverine (Fig. 5). Under the conditions of this experiment, extensive labeling of acceptor sites on the monomeric $\gamma$-chain of normal fibrin had occurred; a small proportion of $\gamma$-chains had also formed fluorescent $\gamma$-dimers (gel 1). Some incorporation of fluorescent label into $\alpha$-chains was also evident and there were some faint, higher molecular weight fluorescent bands, presumably due to incorporation of fluorescent label into $\alpha$-polymers. The Paris I fibrin sample exhibited a fluorescent band in the position of the $\gamma$-chain and the $\alpha$-chain. The intensity of fluorescence in the $\alpha$-chain position relative to that in the $\gamma$-chain position was greater than in normal fibrin, presumably because so many fewer $\gamma$-chains were present in the Paris I fibrin preparation. There was no indication that dansylcadaverine had been incorporated into the $\gamma$ Paris I position.

Plasmic hydrolysis of normal and Paris I fibrinogen. Hydrolysis with plasmin has been used in many investigations to probe the structure of normal fibrinogen (13, $28-32,40-43$ inter alia) as well as that of congenitally abnormal fibrinogen molecules such as fibrinogen Giessen $(44,45)$. Such an analysis was carried out to compare normal and Paris I fibrinogen with respect to the sequence of attack on the molecule and the types of core derivatives formed. Limited amounts of purified Paris I fibrino- gen were available for these studies, thus precluding separation or purification of digest components. Instead, digest samples were examined by electrophoresis in sodium dodecyl sulfate-containing gels (Fig. 6). Digestion was carried out at several enzyme-substrate ratios to examine all phases of hydrolysis. For each experiment however, care was taken to digest normal fibrinogen under the same conditions, so that timed samples of normal and Paris I fibrinogen digests were at an equivalent phase of hydrolysis. The findings demonstrated the evolution of at least one transient form of Fragment D which was present only in the Paris I digests; a description of these events follows.

From hydrolytic stage 1 through early stage 3 , no differences were discernible in the migration rates of unreduced core species (Fig. 6, gels 1-4) ${ }^{2}$ At a somewhat later phase of stage 3 digestion, a band (designated DParis I) appeared in unreduced Paris I digest samples (gel 6) which had no recognizable counterpart in the normal fibrinogen digest (gel 5). Furthermore, material migrating in the $\mathrm{D}_{2}$ position (gel 6) in the Paris I digests was more heterogeneous than that of normal fibrinogen (in some gels at this phase this position could be resolved into a doublet); this finding suggests that more

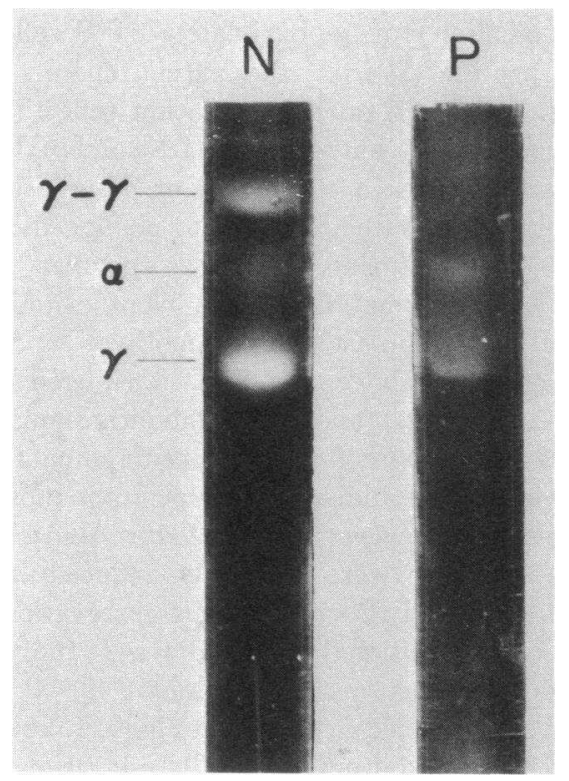

FIGURE 5 Dodecyl sulfate electrophoresis (9\% acrylamide) of reduced samples of normal fibrin $(\mathrm{N})$ and Paris I fibrin (P) subjected to cross-linking in the presence of Factor XIIIa and monodansylcadaverine. Incorporation of the fluorescent amine was detected and photographed under UV light. In order to obtain satisfactory photographs, a relatively heavy protein load of Paris I fibrin was run, this probably accounts for the "trailing" of the $\gamma$-chain fluorescence in gel 2 into the $\gamma$ Paris I region. Lighter protein loads which were visually satisfactory but unsuitable for photography revealed no evidence of trailing. 


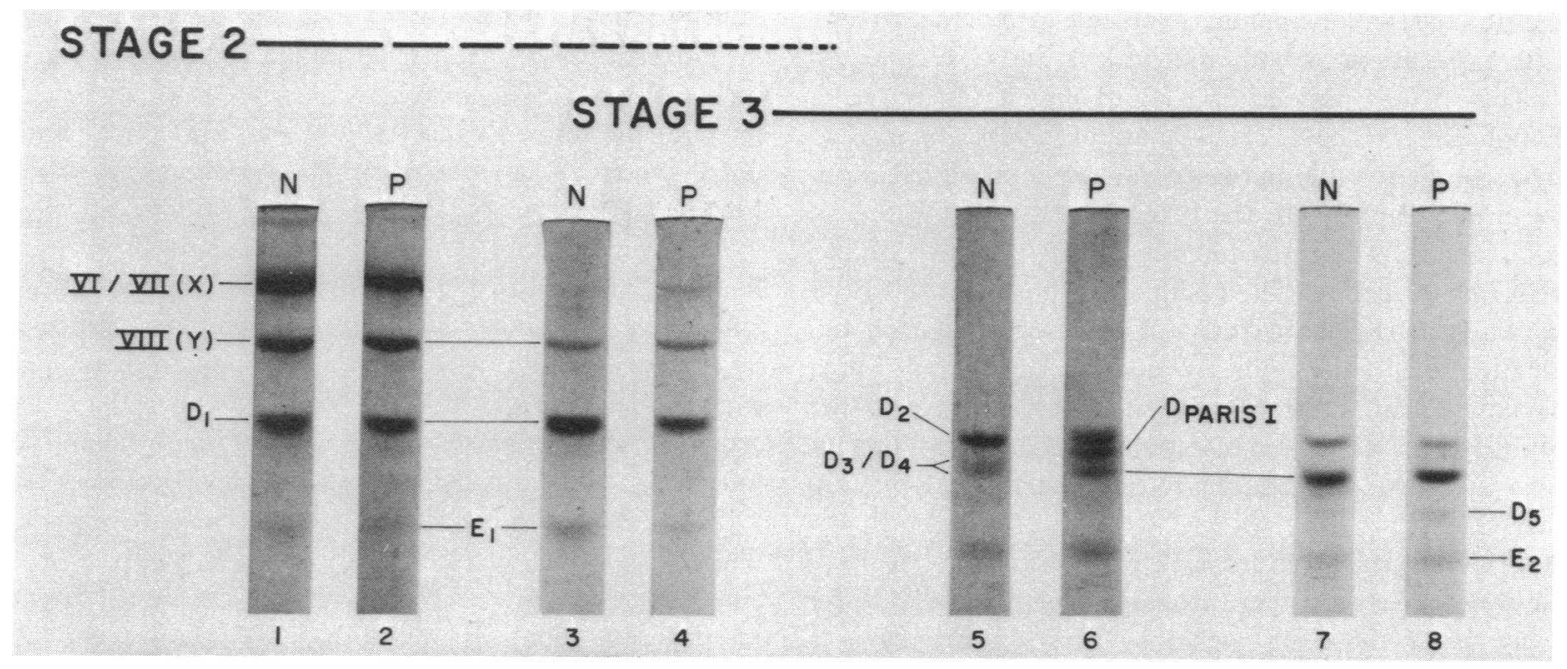

Figure 6 Dodecyl sulfate electrophoresis (5\% acrylamide) of unreduced samples from plasmic digests of normal fibrinogen fraction I-4 (N) and Paris I peak 1 fibrinogen (P). Digestion and sampling for each paired set of gels was carried out under the same conditions. The phases of digestion at which samples were taken are indicated by their positions in the diagram.

than one unique form of Fragment $\mathrm{D}$ may be evolved in Paris I digests. Additional observations include the absence from Paris I digests of material corresponding in position to band $\mathrm{D}_{3}$. At a more advanced digestive phase, the DParis I band as well as the heterogeneity observed in the $\mathrm{D}_{2}$ region were no longer features of the gel samples (gel 8) which, except for a relatively increased staining intensity of the D\& band, became indistinguishable from their normal counterparts.

During the digestive phases described above, Fragment $\mathrm{E}$ also is evolved. As digestion proceeds its size is reduced. At no phase of hydrolysis was there any indication of a difference in the electrophoretic characteristics of Fragment E species derived from Paris I digests and those generated from normal fibrinogen.

Gradient elution chromatography of normal and Paris I fibrinogen. DEAE-cellulose gradient elution chromatography (four chromatograms of two preparations) of Paris I fraction I-2 was carried out (Fig. 1) to characterize the preparations and as a purification procedure (see Methods). Highly purified normal fibrinogen (fraction I-4) ordinarily exhibits two major fibrinogen peaks ; less purified preparations (such as fraction I-2) also exhibit a third minor fibrinogen peak (Fig. 1. tube 6061) and another later eluting peak (tube 71-78) containing nonfibrinogen proteins such as cold-insoluble globulin (9). Analyses of normal fibrinogen chains (46) have indicated that the differences in elution characteristics of peaks 1 and 2 fibrinogen can be accounted for by the absence (peak 1) or the presence (peak 2) of a $\gamma$-chain variant termed $\gamma^{\prime}$. Paris I fibrinogen, like normal, exhibited two major fibrinogen-containing peaks.
However, the Paris I fibrinogen peaks emerged later than their presumed normal counterparts. After correction for peak overlap, it was estimated that $80 \%$ of the fibrinogen-related material in the normal fraction I-2 chromatogram was eluted as peak 1 , the remainder eluting in peaks 2 and 3 . The Paris I fibrinogen chromatogram exhibited a similar distribution, since its peak 1 fibrinogen amounted to $81 \%$ of the total.

In three of five chromatograms, as illustrated by Fig. 1, there was a slight "shoulder" on the ascending limb of peak 1, suggesting the possible presence of a population of normal fibrinogen molecules (i.e. having two $\gamma$-chains). Its presence was confirmed by demonstrating (data not shown) that material obtained from the ascending limb of peak 1 has a considerably higher proportion of $\gamma$-chains than of $\gamma$ Paris I chains.

\section{DISCUSSION}

Based upon previous experiments with samples of fibrinogen Baltimore (47), which also manifested delayed elution of chromatographic peaks 1 and 2. it appears that the amount of normal fibrinogen emerging in the ascending portion of Paris I fibrinogen peak 1 does not amount to more than $10 \%$ of the total fibrinogen population in the preparations we have analyzed. This value seems reasonable in view of the preponderance of $\gamma$ Paris I chains in all plasma subfractions examined. However, since we have not examined fibrinogen of relatively high solubility (i.e. not precipitated in Cohn fraction I), we cannot yet make a final assessment of the proportion of the total fibrinogen which is normal. 
The $\gamma$ Paris I chains comprise a high proportion of the $\gamma$-chain population in both the peak 1 and 2 fibrinogen pools ( $65-75 \%$ of peak $1 ; 67-82 \%$ of peak 2 fibrinogen). Although the presence of the mutant chain almost cer tainly accounts for the increased anionic-binding behavior of the preparation itself, the heterogeneity manifested as peaks 1 and 2 is not attributable to the presence of the $\gamma$ Paris I chain per se. The results are consistent with the view that the region of the $\gamma$-chain accounting for the chromatographic behavior of peak 2 fibrinogen (viz., $\gamma^{\prime}$-variant chain, reference 46) is present in both the $\gamma$ - and $\gamma$ Paris I chain populations. Thus, this property of $\gamma$ Paris I chains suggests that it has structural overlap with $\gamma$-chains.

Apart from its apparent lack of a thrombin- or Reptilase-sensitive site, other properties of $\gamma$ Paris I differ sharply from those of $\gamma$-chains. First, $\gamma$ Paris I is a larger chain than $\gamma$, and this by itself permits it to be distinguishable in dodecyl sulfate gel-electrophoretic experiments (reference 8 , this study). Second, unlike a $\gamma$-chain, the $\gamma$ Paris I chain does not participate in $\gamma$-dimer formation (reference 8 , this study); it also fails to incorporate monodansylcadaverine in the presence of Factor XIIIa (Fig. 5). These latter two observations indicate that the amine acceptor site in the $\mathrm{COOH}$-terminal region of the $\gamma$-chain (36) is either not present on this mutant chain or is unavailable ${ }^{3}$ for cross-linking. Further support for the localization of the abnormality in the $\mathrm{COOH}$-terminal region of the molecule is provided from examination of the products of plasmic digestion. That is, plasmic Fragment $\mathrm{E}$ derived from Paris I fibrinogen does not differ from normal, whereas a unique form of Fragment D (DParis I) has been identified in Paris I digests (Fig. 6). Fragment $\mathrm{E}$ consists only of structures derived from its $\mathrm{NH}_{2}$-terminal region, whereas Fragment $\mathrm{D}$ consists of chain fragments whose structure overlaps that of the $\mathrm{COOH}$-terminal region of the molecule (13, 32, 40-43 inter alia).

In contrast to the abnormality demonstrated in the $\gamma$ Paris I chain, when scrutinized from the standpoint of their electrophoretic migration rates, and functional qualities upon treatment with Reptilase or thrombin, the $\mathrm{A} \alpha-, \mathrm{B} \beta$-, and $\gamma$-chain populations of fibrinogen Paris I are indistinguishable from those in normal fibrinogen. The $\gamma$-chains however, are reduced in amount. The demonstration of delayed $\gamma$-dimer and $\alpha$-polymer formation of Paris I fibrin is not attributable to an intrinsic abnor-

\footnotetext{
${ }^{3}$ Steric availability of such a site is an important consideration. Only one glutaminyl residue on the $\boldsymbol{\gamma}$-chain participates in cross-linking (36), although several are present in the $\mathrm{COOH}$-terminal region. Despite the fact that $\beta$-chains of fibrin do not participate in cross-linking $(37,38,48)$ and fail to incorporate monodansylcadaverine in the presence of Factor XIIIa (27), $S$-sulfo- $\beta$-chains can undergo considerable cross-linking (49).
}

mality of these chains themselves since cross-linking of normal fibrin is also impaired in the presence of Paris I fibrin (Fig. 4). The relationship between this phenomenon and the known capacity of Paris I fibrin to impair aggregation of normal fibrin (4) remains to be established.

Substitution of an elongated mutant chain for a normal chain is unique as far as congenital abnormalities of fibrinogen are concerned, but such an occurrence has several precedents with respect to human hemoglobin variants. For example, hemoglobin mutants have been reported which manifest elongation of the $\alpha$-chains (hemoglobin Constant Spring (50); hemoglobin Wayne (51) ; hemoglobin Icaria (52) or $\beta$-chains (hemoglobin Tak, 53). These elongations all occur by addition of peptide material at the $\mathrm{COOH}$-terminus and are thought to be brought about by mutation of a chain-terminating codon $(50,52,53)$ or by a "frame-shift" (51). It may be useful to draw attention to the fact that available information for the $\gamma$ Paris I mutant also points to an abnormality in the $\mathrm{COOH}$-terminal region.

\section{ACKNOWLEDGMENTS}

We are grateful to the medical illustrations/photography section at Downstate Medical Center for their cooperation in developing the figures for this work. We are also indebted to Miss Lori Lefkowitz for her excellent secretarial services.

This research was supported by grants from the National Heart and Lung Institute (HL-11409 and HL-17419), Institut National de la Santé et de la Recherche Médicale, ATP 12-74-33, and UER Xavier-Bichat.

\section{REFERENCES}

1. Ménaché, D. 1964. Constitutional and familial abnormal fibrinogen. Thromb. Diath. Hacmorrh. (Suppl.) 13: 173-185.

2. Ménaché, D. 1973. Abnormal fibrinogens. A review. Thromb. Diath. Haemorrh. 29 : 525-535.

3. Mammen, E. F. 1974. Congenital abnormalities of the fibrinogen molecule. Semin. Thromb. Hemost. 1: 184 201.

4. Guillin, M-C., and D. Ménaché. 1973. Fetal fibrinogen and fibrinogen Paris I: Comparative fibrin monomers aggregation studies. Thromb. Res. 3: 117-135.

5. Blombäck, B. 1970. In discussion. Thromb. Diath. Hacmorrh. (Suppl.) $39: 337$.

6. Blombäck, B., and M. Blombäck. 1970. Molecular defects 671-678.

and variants of fibrinogen. Nour. Rev. Fr. Hématol. 10:

7. Mosesson, M. W. 1970. In discussion. Throm. Diath. Hacmorrh. (Suppl.) 39: 337-338.

8. Budzynski, A. Z., V. J. Marder, D. Ménaché, and M-C. Guillin. 1974. Defect in the gamma polypeptide chain of a congenital abnormal fibrinogen (Paris I). Nature (Lond.). 252: 66-68.

9. Mosesson, M. W., and R. A. Umfleet. 1970. The coldinsoluble globulin of human plasma. I. Purification, primary characterization, and relationship to fibrinogen and other cold-insoluble fraction components. J. Biol. Chem. 245 : 5728-5736. 
10. Mosesson, M. W., N. Alkjaersig, B. Sweet, and S. Sherry. 1967. Human fibrinogen of relatively high solubility. Comparative biophysical, biochemical and biological studies with fibrinogen of lower solubility. Biochemistry. $6: 3279-3287$.

11. Weber, K., and M. Osborn. 1969. The reliability of molecular weight determinations by dodecyl sulfatepolyacrylamide gel electrophoresis. J. Biol. Chem. 244: 4406-4412.

12. Mosesson, M. W., J. S. Finlayson, R. A. Umfleet, and D. Galanakis. 1972. Human fibrinogen heterogeneities. I. Structural and related studies of plasma fibrinogens which are high solubility catabolic intermediates. $J$. Biol. Chem. 247: 5210-5219.

13. Mosesson, M. W., J. S. Finlayson, and D. K. Galanakis. 1973. The essential covalent structure of human fibrinogen evinced by analysis of derivatives formed during plasmic hydrolysis. J. Biol. Chem. 248: 7913-7929.

14. Scheidegger, J. J. 1955. Une micro-méthode de l'immuno-électrophorèse. Int. Arch. Allergy Appl. Immunol. 7: 103-110.

15. Laurell, C-B. 1972. Electroimmuno assay. Scand. J. Clin. Lab. Invest. 29: (Suppl. 124) : 21-37.

16. $\mathrm{McF}$ arlane, A. S. 1963. In vivo behavior of $\mathrm{I}^{181}$-fibrinogen. J. Clin. Invest. 42: 346-361.

17. Krohn, K., L. Sherman, and M. Welch. 1972. Studies of radioiodinated fibrinogen. I. Physiochemical properties of the $\mathrm{ICl}$, chloramine-T, and electrolytic reaction products. Biochim. Biophys. Acta. 285: 404-413.

18. Ratnoff, O. D., and C. Menzie. 1951. A new method for the determination of fibrinogen in small samples of plasma. J. Lab. Clin. Med. 37: 316-320.

19. Cohn, E. J., L. E. Strong, W. L. Hughes, Jr., D. J. Mulford, J. N. Ashworth, M. Melin, and H. L. Taylor. 1946. Preparation and properties of serum and plasma proteins. IV. A system for the separation into fractions of the protein and lipoprotein components of biological tissues and fluids. J. Am. Chem. Soc. 68: 459475.

20. Ménaché, D. 1970. Congenitally abnormal fibrinogens. Thromb. Diath. Haemorrh. (Suppl.) 39: 307-321.

21. Blombäck, B., and M. Blombäck. 1956. Purification of human and bovine fibrinogen. Ark. Kemi. 10: 415-443.

22. Laki, K. 1951. The polymerization of proteins: the action of thrombin on fibrinogen. Arch. Biochem. 32: 317-324.

23. Mosesson, M. W., and S. Sherry. 1966. The preparation and properties of human fibrinogen of relatively high solubility. Biochemistry. 5 : 2829-2835.

24. Finlayson, J. S., and M. W. Mosesson. 1963. Heterogeneity of human fibrinogen. Biochemistry. 2: 42-46.

25. Loewy, A. G., K. Dunathan, R. Kriel, and H. L. Wolfinger, Jr. 1961. Fibrinase I. Purification of substrate and enzyme. J. Biol. Chem. 236: 2625-2633.

26. Chung, S. I., and J. E. Folk. 1972. Kinetic studies with transglutaminases. The human blood enzymes (activated coagulation factor XIII) and the guinea pig hair follicle enzyme. J. Biol. Chem. 247: 2798-2807.

27. Lorand, L., D. Chenoweth, and A. Gray. 1972. Titration of the acceptor cross-linking sites in fibrin. Ann. N. Y. Acad. Sci. 202: 155-171.

28. Marder, V. J., N. R. Shulman, and W. R. Carroll. 1969. High molecular weight derivatives of human fibrinogen produced by plasmin. I. Physicochemical and immunological characterization. J. Biol. Chem. 244: 2111-2119.
29. Nussenzweig, V., M. Seligmann, J. Pelmont, and P. Grabar. 1961. Les Produits de dégradation du fibrinogène humain par la plasmine. I. Séparation et propriétés physico-chimiques. Ann. Inst. Pasteur Paris. 100: 377389.

30. Mills, D., and S. Karpatkin. 1972. The initial macromolecular derivatives of human fibrinogen produced by plasmin. Biochim. Biophys. Acta. 271: 163-173.

31. Mosesson, M. W., D. K. Galanakis, and J. S. Finlayson. 1974. Comparison of human plasma fibrinogen subfractions and early plasmic fibrinogen derivatives. $J$. Biol. Chem. 249: 4656-4664.

32. Marder, V. J., A. Z. Budzynski, and H. L. James, 1972. High molecular weight derivatives of human fibrinogen produced by plasmin. III. Their $\mathrm{NH}_{2}$-terminal amino acids and comparison with the " $\mathrm{NH}_{2}$-terminal disulfide knot." J. Biol. Chem. 247 : 4775-4781.

33. Sgouris, J. T., J. K. Inman, K. B. McCall, L. A. Hyndman, and H. D. Anderson. 1960. The preparation of human fibrinolysin (plasmin). Vox Sang. 5: 357-376.

34. Matačić, S., and A. G. Loewy. 1968. The identification of isopeptide crosslinks in insoluble fibrin. Biochem. Biophys. Res. Commun. 30: 356-362.

35. Pisano, J. J., J. S. Finlayson, and M. P. Peyton. 1968. Cross-link in fibrin polymerized by Factor XIII: $\epsilon-(\gamma-$ glutamyl)lysine. Science (Wash. D. C.). 160: 892-893.

36. Chen, R., and R. F. Doolittle. 1971. $\gamma-\gamma$ Cross-linking sites in human and bovine fibrin. Biochemistry. 10: 4486-4491.

37. McKee, P. A., P. Mattock, and R. L. Hill. 1970. Subunit structure of human fibrinogen, soluble fibrin, and cross-linked insoluble fibrin. Proc. Natl. Acad. Sci. U. S. A. 66: 738-744.

38. Takagi, T., and S. Iwanaga. 1970. Polypeptide chain involved in the cross-linking of stabilized bovine fibrin. Biochem. Biophy's. Res. Commun. 38: 129-136.

39. McDonagh, R. P., Jr., J. McDonagh, M. Blombäck, and B. Blombäck. 1971. Crosslinking of human fibrin: evidence for intermolecular crosslinking involving $\alpha$ chains. FEBS (Fed. Eur. Biochem. Soc.) Lett. 14: 3336.

40. Pizzo, S. V., M. L. Schwartz, R. L. Hill, and P. A. McKee. 1972. The effect of plasmin on the subunit structure of human fibrinogen. J. Biol. Chem. 247: 636645.

41. Furlan, M., and E. A. Beck. 1972. Plasmin degradation of human fibrinogen. I. Structural characterization of degradation products. Biochim. Biophys. Acta. 263: 631-644.

42. Mills, D. A. 1972. A molecular model for the proteolysis of human fibrinogen by plasmin. Biochim. Biophys. Acta. 263: 619-630.

43. Budzynski, A. Z., V. J. Marder, and J. R. Shainoff. 1974. Structure of plasmic degradation products of human fibrinogen. Fibrinopeptide and polypeptide chain analysis. J. Biol. Chem. 249: 2294-2302.

44. Krause, W. H., D. L. Heene, and H. G. Lasch. 1973. Congenital dysfibrinogenemia (fibrinogen Giessen). Thromb. Diath. Haemorrh. 29: 547-561.

45. Krause, W. H., and H. Bleyl. 1973. Investigation on plasmin-proteolysis of abnormal fibrinogen (fibrinogen Giessen). Thromb. Res. 2: 361-364.

46. Mosesson, M. W., J. S. Finlayson, and R. A. Umfleet. 1972. Human fibrinogen heterogeneities. III. Identification of $\gamma$ chain variants. J. Biol. Chem. 247: 5223-5227. 
47. Mosesson, M. W., and E. A. Beck. 1969. Chromatographic, ultracentrifugal, and related studies of fibrinogen "Baltimore." J. Clin. Invest. 48: 1656-1662.

48. Chen, R., and R. F. Doolittle. 1969. Identification of the polypeptide chains involved in the cross-linking of fibrin. Proc. Natl. Acad. Sci. U. S. A. 63: 420-427.

49. Finlayson, J. S., M. W. Mosesson, T. J. Bronzert, and J. J. Pisano. 1972. Human fibrinogen heterogeneities. II. Cross-linking capacity of high solubility catabolic intermediates. J. Biol. Chem. 247: 5220-5222.

50. Clegg, J. B., D. J. Weatherall, and P. F. Milner. 1971. Haemoglobin Constant Spring-a chain termination mutant? Nature (Lond.). 234: 337-340.
51. Seid-Akhavan, M., W. P. Winter, R. K. Abramson, and D. L. Rucknagel. 1972. Hemoglobin Wayne: A frameshift variant occurring in two distinct forms. Blood. 40: 927.

52. Clegg, J. B., D. J. Weatherall, I. Contopolou-Griva, K. Caroutsos, P. Poungouras, and H. Tsevrenis. 1974. Haemoglobin Icaria, a new chain-termination mutant which causes $\alpha$ thalassaemia. Nature (Lond.). 251: 245-247.

53. Flatz, G., J. L. Kinderlerer, J. V. Kilmartin, and H. Lehmann. 1971. Haemoglobin Tak: A variant with additional residues at the end of the $\beta$-chains. Lancet. $1: 732-733$. 